\title{
Mass burning rate of premixed stretched flames: integral analysis versus large-activation-energy asymptotics
}

\author{
L. P. H. de Goey · J. H. M. ten Thije Boonkkamp
}

Received: 20 December 2006 / Accepted: 11 July 2007 / Published online: 22 August 2007

(C) Springer Science + Business Media B.V. 2007

\begin{abstract}
New expressions for the mass burning rate are derived from a recently introduced flamelet model using integral analysis. The results are compared with well-known expressions, based on large-activation-energy asymptotics. There is no restriction on Lewis numbers and the expressions reduce to the same results as found earlier with asymptotic techniques for Lewis numbers close to 1 . From our analysis it appears that the burned edge of a stretched flamelet is most appropriate to determine the mass burning rate. The consequences for experimental and numerical studies are investigated.
\end{abstract}

Keywords Flamelet model · Flat stagnation flame $\cdot$ Karlovitz integral $\cdot$ Markstein number · Mass burning rate

\section{Introduction}

In gas mixtures of fuel and oxydizer (air), combustion generally takes place in thin layers, i.e., premixed flame fronts propagate relative to the unburned mixture with a well-defined velocity, referred to as the laminar burning velocity. Its magnitude is called the laminar burning speed $s_{\mathrm{L}}$ and is a key parameter in combustion science. The hot, reacted gases preheat the unburned mixture at the leading-edge molecular-diffusion processes (preheat zone) up to a point where the temperature has increased to a high-enough value that chemical reactions of the combustion process can take place (reaction zone). This reaction zone is usually much thinner than the preheat zone, which is generally of the order of $1 \mathrm{~mm}$ for hydrocarbon-air flames. If the flame front has a planar shape and the flow enters the front as a laminar, homogeneous plug-type flow, the one-dimensional system propagates with the laminar burning speed $s_{\mathrm{L}}^{0}$ relative to the unburned mixture. In general, however, it is very difficult to create this situation in practice. Flames often display curvature effects and the flow is often strained ahead of the flame, e.g., due to the burner geometry or turbulent structures in the flow. This leads effectively to what is referred to as flame-stretch. The flame-stretch

L. P. H. de Goey

Department of Mechanical Engineering, Eindhoven University of Technology, P.O. Box 513, Eindhoven 5600 MB,

The Netherlands

J. H. M. ten Thije Boonkkamp ( $\varangle)$

Department of Mathematics and Computer Science, Eindhoven University of Technology, P.O. Box 513, Eindhoven 5600 MB,

The Netherlands

e-mail: tenthije@win.tue.nl 
rate is a parameter which describes the relative rate of change of flame surface area due to flame curvature, flow straining and flame-propagation effects.

It is well known that flame stretch plays an important role in the propagation, stability and structure of premixed flames, because the burning speed is sensitive to the local stretch rate. Stretch effects were first studied by Karlovitz et al. [1], Lewis and von Elbe [2, Sect. V.5 and V.6] and Markstein [3, Chapt. C] to investigate flame extinction, flame stabilization and flame-front instability. Since these early publications, significant progress has been made in the understanding of flame stretch and in particular the structure, propagation and dynamics of stretched flames has been studied in numerous papers using large-activation-energy-asymptotics (LAEA); see e.g. [4-6]. Clavin [7] and Clavin and Williams [8] rigorously derived expressions for the mass burning rate using LAEA for flames described by simple chemistry and Lewis numbers close to 1 . This theory has been used, for instance, in laminar flamelet models for turbulent premixed flames; see e.g. [9]. Chung and Law [10] used another approach, the integral analysis (IA), to study stretched flames. Identical phenomena are predicted with their theory, although the resulting expressions are different. The LAEA and IA theories have never been compared thoroughly so far.

Using the IA rigorously, de Goey et al. [11-13] and ten Thije Boonkkamp et al. [14,15] studied the mass burning rate of stretched flames with multiple-species transport and chemistry. They introduced a mass-based stretch field for spatially resolved flames and derived expressions for the mass burning rate, containing integrals of the stretch field, describing the flame response. There is no restriction on the Lewis numbers. In the present paper we will use this approach to show that LAEA results, first found by Joulin and Clavin [16], are recovered with the IA method to lowest order in the Zeldovich number for flat, weakly strained flames when density variations are neglected and for Lewis numbers close to 1 . Furthermore, the same results as derived by Clavin and Williams [7,8] are found to lowest order in the Zeldovich number when density variations due to gas expansion are included, again for Lewis numbers close to 1. Finally, from a theoretical and numerical investigation of a spatially resolved stretched flame, it is shown that the mass burning rate at the burned edge of the flame is most accurate for describing flame propagation. This is very important for a quantitative interpretation of experimental and numerical data of stretched flames. If a position different from the burned flame edge is chosen to derive the mass burning rate, correction terms have to be used, which are also derived in this paper.

The paper is organized as follows. In Sect. 2, we summarize the flamelet model of de Goey and ten Thije Boonkkamp. General expressions for the mass burning rate $m_{\mathrm{b}}$ in the burned gases of stretched flames are presented in Sect. 3. These expressions have been applied successfully in the last few years to analyze and describe strong stretch effects in turbulent flames for unit Lewis numbers [17] and in weakly stretched flames governed by multiplespecies chemistry and transport with non-unit Lewis numbers [18]. In Sect. 4, we study the mass burning rate $m_{\mathrm{b}}$ for the case of flat weakly strained stagnation flames without gas expansion, described by a one-step irreversible reaction. Variations in the density caused by gas expansion are studied in the subsequent section. The mass burning rate $m_{\mathrm{u}}$ in the unburned gases is studied in Sect. 6. Different results are found for Markstein numbers in either burned or unburned gases. In Sect. 7 our analysis shows that Markstein numbers for the burned gases previously found by Joulin and Clavin [16] and Clavin [8] are reproduced for Lewis numbers close to 1. For the unburned gases, the Markstein number of Clavin and Williams [7] is also recovered for Lewis numbers close to 1. A clear physical picture of how $m_{\mathrm{u}}$ and $m_{\mathrm{b}}$ are to be interpreted inside the flame structure is also presented in Sect. 7.

\section{Flamelet model for premixed stretched flames}

In this section we summarize the flamelet model for premixed, stretched flames, as introduced in our previous papers $[11,12,14]$.

We define a flame as a region in space where a suitable progress variable $\mathcal{Y}$ assumes values between the unburned $\left(\mathcal{Y}_{\mathrm{u}}\right)$ and burned $\left(\mathcal{Y}_{\mathrm{b}}\right)$ values, in other words, it consists of the preheat zone and the reaction zone. For $\mathcal{Y}$ we may take, e.g., one of the species mass fractions $Y_{i}$ or the temperature $T$, provided $\nabla \mathcal{Y} \neq \mathbf{0}$ everywhere in the flame. In this paper we choose $\mathcal{Y}=T$. We introduce flame surfaces as isotherms, i.e., surfaces where $T(\boldsymbol{x}, t)=$ Const, and define a curvilinear coordinate system $\xi=\left(\xi^{1}, \xi^{2}, \xi^{3}\right)$ by the relations 
$T\left(x_{1}, x_{2}, x_{3}, t\right)=T\left(\xi^{1}\right), \quad \frac{\partial \boldsymbol{x}}{\partial \xi^{1}} \cdot \frac{\partial \boldsymbol{x}}{\partial \xi^{\alpha}}=0 \quad(\alpha=2,3) \quad$ for $\quad T_{\mathrm{u}} \leq T \leq T_{\mathrm{b}}$,

i.e., the coordinate surfaces $\xi^{1}=$ Const coincide with flame surfaces and the $\xi^{1}$-coordinate lines are orthogonal to the $\xi^{\alpha}$-coordinate lines $(\alpha=2,3)$. Note that the $\xi^{1}$-coordinate is not uniquely defined by the relations in (2.1), since these also hold for $f\left(\xi^{1}\right)$ with $f$ a sufficiently smooth and monotone function. In each flame surface, $\left(\xi^{2}, \xi^{3}\right)$ is a curvilinear coordinate system, not necessarily orthogonal, which will not be further specified. For timedependent flames, the flame surfaces will move in the spatial domain with velocity $\boldsymbol{v}_{\mathrm{f}}=\boldsymbol{v}+s_{\mathrm{L}} \boldsymbol{n}$, where $\boldsymbol{v}$ is the flow velocity, $s_{\mathrm{L}}$ the laminar burning speed and $\boldsymbol{n}$ the unit normal directed towards the unburned gas mixture, resulting in a time-dependent coordinate system $\xi=\xi(x, t)$. Moreover, we introduce the variable $\tau$ as the time corresponding to the curvilinear coordinate system. Obviously, $\tau=t$.

In the flamelet model that follows, we use the mass-based definition of the stretch rate $K$ [13], which is an extension of the usual definition [19] and applicable to flames of finite thickness. It is defined as

$K:=\frac{1}{M} \frac{\mathrm{d} M}{\mathrm{~d} t}$ with $M(t):=\int_{\Omega(t)} \rho \mathrm{d} V$,

where $\mathrm{d} / \mathrm{d} t:=\partial / \partial t+\boldsymbol{v}_{\mathrm{f}} \cdot \nabla$ is the time derivative when following the flame surfaces and where $\rho$ is the density of the gas mixture, i.e., $K$ is the fractional rate of change of the mass $M(t)$ contained in a small volume $\Omega(t)$ in the flame, moving with velocity $\boldsymbol{v}_{\mathrm{f}}$. Applying the Reynolds transport theorem [20, pp. 84-85] to (2.2), we obtain the following expression for $K$ :

$\rho K=\frac{\partial \rho}{\partial t}+\nabla \cdot\left(\rho \boldsymbol{v}_{\mathrm{f}}\right)$

Formulated in $(\xi, \tau)$-variables, the expression for $K$ reads

$K=\frac{1}{\sqrt{g}} \frac{\partial}{\partial \tau}(\sqrt{g})+\frac{1}{\rho} \frac{\partial \rho}{\partial \tau}$,

where $\sqrt{g}$ is the Jacobian of the coordinate transformation $x \mapsto \xi$, given by

$\sqrt{g}=h_{1} \sigma, \quad h_{1}:=\left|\frac{\partial \boldsymbol{x}}{\partial \xi^{1}}\right|, \quad \sigma:=\left|\frac{\partial \boldsymbol{x}}{\partial \xi^{2}} \times \frac{\partial \boldsymbol{x}}{\partial \xi^{3}}\right|$.

Clearly, $\mathrm{d} V:=\sqrt{g} \mathrm{~d} \xi^{1} \mathrm{~d} \xi^{2} \mathrm{~d} \xi^{3}$ is the volume element in the $\xi$-coordinate system, $\mathrm{d} S:=\sigma \mathrm{d} \xi^{2} \mathrm{~d} \xi^{3}$ is the area element on a flame surface $\xi^{1}=$ Const and $\mathrm{d} s:=h_{1} \mathrm{~d} \xi^{1}$ is the arc-length element along a $\xi^{1}$-coordinate line. Apart from the usual terms related to flame curvature and flow straining, relation (2.4) for $K$ incorporates additional terms, e.g., due to flame thickness variations. Note that $K$ is a scalar field, defined in the entire flame zone, whereas in other theories the stretch rate is restricted to a single flame surface.

The set of governing equations for premixed flames can be classified in the following three groups, i.e., first, the flamelet equations for the combustion variables $Y_{i}, T$ and the mass burning rate $m:=\rho s_{\mathrm{L}}$, describing transport and chemistry in the flame, second, the momentum equations coupled with the flamelet equations for the specific enthalpy $h$ and element mass fractions $Z_{j}$, describing flow and mixing without chemical reactions, and third, a kinematic equation describing the motion of the flame. We have derived the first system of equations by rewriting the corresponding conservation equations in terms of the curvilinear coordinate system $\boldsymbol{\xi}$ and inserting expression (2.3) for the stretch rate; for more details see e.g. $[12,21]$. This way we obtain the following set of quasi-one-dimensional flamelet equations 


$$
\begin{aligned}
& \frac{\partial}{\partial s}(\sigma m)=-\sigma \rho K, \\
& \frac{\partial}{\partial s}\left(\sigma m Y_{i}\right)-\frac{1}{\operatorname{Le}_{i}} \frac{\partial}{\partial s}\left(\sigma \frac{\lambda}{c_{p}} \frac{\partial Y_{i}}{\partial s}\right)=\sigma\left(\omega_{i}-\rho K Y_{i}\right), \quad\left(i=1, \ldots, N_{\mathrm{s}}\right), \\
& \frac{\partial}{\partial s}(\sigma m T)-\frac{1}{c_{p}} \frac{\partial}{\partial s}\left(\sigma \lambda \frac{\partial T}{\partial s}\right)=\sigma\left(\omega_{T}-\rho K T\right), \quad \omega_{T}:=\frac{-1}{c_{p}} \sum_{i=1}^{N_{\mathrm{s}}} h_{i} \omega_{i} .
\end{aligned}
$$

Other variables/parameters in (2.6) are the number of different species $N_{\mathrm{s}}$, the species reactions rates $\omega_{i}$, the temperature source term $\omega_{T}$, the thermal conductivity $\lambda$, the specific heat (at constant pressure) $c_{p}$ and the Lewis numbers $\mathrm{Le}_{i}$ (assumed to be constant). In the derivation of (2.6) we have assumed that conduction and diffusion fluxes along the flame surfaces are negligible compared to the stretch terms, proportional to $K$, which is justified in the so-called flamelet combustion regime where chemistry is fast compared to the flow time scales. We further assumed that all specific heats are equal and constant, i.e., $c_{p, i}=c_{p}$. The set has to be closed with an equation of state, which for constant ambient pressure $p_{\mathrm{amb}}$, describing low-Mach-number deflagrations, may be written as

$$
\frac{\rho R T}{W}=p_{\mathrm{amb}}, \quad \frac{1}{W}=\sum_{i=1}^{N_{\mathrm{s}}} \frac{Y_{i}}{W_{\mathrm{s}, i}}
$$

with $R$ the universal gas constant, $W$ the mean molecular weight of the mixture and $W_{\mathrm{s}, i}$ the species molecular weights.

Since the mass burning rate depends on the enthalpy and element composition in the reaction layer [12], we have to take into account the flamelet equations for the specific enthalpy $h$ and the mass fractions $Z_{j}$ of the $N_{\mathrm{e}}$ elements involved. Recall, that these variables are defined by

$h:=\sum_{i=1}^{N_{\mathrm{s}}} h_{i} Y_{i}, \quad h_{i}=h_{i, \mathrm{ref}}+\int_{T_{\mathrm{ref}}}^{T} c_{p, i}\left(T^{\prime}\right) \mathrm{d} T^{\prime}, \quad Z_{j}:=\sum_{i=1}^{N_{\mathrm{s}}} w_{j, i} Y_{i}, \quad\left(j=1, \ldots, N_{\mathrm{e}}\right)$,

where $h_{i}, h_{i, \text { ref }}$ and $c_{p, i}$ are the specific enthalpy, the specific enthalpy of formation at reference temperature $T_{\text {ref }}$ and the specific heat (at constant pressure), respectively, of species $i$. Moreover, the coefficients $w_{i, j}$ are defined by $w_{j, i}:=W_{\mathrm{e}, j} \mu_{j, i} / W_{\mathrm{s}, i}$, with $W_{\mathrm{e}, j}$ the molecular weight of element $j$ and $\mu_{j, i}$ the number of atoms of element $j$ in species $i$. Analogous to the derivation of (2.6) we find

$$
\begin{aligned}
& \frac{\partial}{\partial s}(\sigma m h)-\frac{\partial}{\partial s}\left(\sigma \frac{\lambda}{c_{p}} \frac{\partial h}{\partial s}\right)-\sum_{i=1}^{N_{\mathrm{s}}}\left(\frac{1}{\mathrm{Le}_{i}}-1\right) \frac{\partial}{\partial s}\left(\sigma \frac{\lambda}{c_{p}} h_{i} \frac{\partial Y_{i}}{\partial s}\right)=-\sigma \rho K h, \\
& \frac{\partial}{\partial s}\left(\sigma m Z_{j}\right)-\frac{\partial}{\partial s}\left(\sigma \frac{\lambda}{c_{p}} \frac{\partial Z_{j}}{\partial s}\right)-\sum_{i=1}^{N_{\mathrm{s}}}\left(\frac{1}{\mathrm{Le}_{i}}-1\right) w_{j, i} \frac{\partial}{\partial s}\left(\sigma \frac{\lambda}{c_{p}} \frac{\partial Y_{i}}{\partial s}\right)=-\sigma \rho K Z_{j}, \quad\left(j=1, \ldots, N_{\mathrm{e}}\right) .
\end{aligned}
$$

Note that the flamelet equations (2.9) do not contain a chemical source term, and for that reason we refer to $h$ and $Z_{j}$ as the conserved variables. Moreover, the set of equations (2.9b) is of course not independent of (2.6b).

\section{Mass burning rate: the general case}

We derive a general expression for the mass burning rate $m_{\mathrm{b}}$ at the burned side of the flame. This mass burning rate depends on the conserved variables $\psi:=\left(h, Z_{j}\right)$ in the burned mixture, which we therefore determine first from the corresponding flamelet equations. 
Suppose, $\sigma=\sigma(s)$ and $K=K(s)$ are given along an $s$-coordinate line. Integrating the flamelet equations (2.6a) and (2.9) across the flame, we obtain the following integral balances

$(\sigma m)_{\mathrm{b}}-(\sigma m)_{\mathrm{u}}=-\int_{s_{\mathrm{u}}}^{s_{\mathrm{b}}} \sigma(s) \rho(s) K(s) \mathrm{d} s$,
$(\sigma m)_{\mathrm{b}}\left(h_{\mathrm{b}}-h_{\mathrm{u}}\right)=-\int_{s_{\mathrm{u}}}^{s_{\mathrm{b}}} \sigma(s) \rho(s) K(s)\left(h(s)-h_{\mathrm{u}}\right) \mathrm{d} s$,
$(\sigma m)_{\mathrm{u}}\left(Z_{j, \mathrm{~b}}-Z_{j, \mathrm{u}}\right)=-\int_{s_{\mathrm{u}}}^{s_{\mathrm{b}}} \sigma(s) \rho(s) K(s)\left(Z_{j}(s)-Z_{j, \mathrm{u}}\right) \mathrm{d} s, \quad\left(j=1, \ldots, N_{\mathrm{e}}\right)$,

where we have assumed that all diffusive/conductive fluxes vanish at the unburned (at $s=s_{\mathrm{u}}$ ) and burned (at $s=s_{\mathrm{b}}$ ) sides of the flame. The subscripts $\mathrm{b}$ and $\mathrm{u}$ denote the value of the corresponding variable in the burned and unburned gas, respectively. For the special case of a stretchless flame, i.e., $K=0$, we obtain $h_{\mathrm{b}}^{0}=h_{\mathrm{u}}^{0}$ and $Z_{j, \mathrm{~b}}^{0}=Z_{j, \mathrm{u}}^{0}\left(j=1, \ldots, N_{\mathrm{e}}\right)$, where the superscript 0 indicates that the variable is considered to be the solution of the corresponding stretchless flamelet equation. Using the expressions for $h$ and $Z_{j}$ in (2.8), we can derive the following formulae for $\Delta h:=h_{\mathrm{b}}-h_{\mathrm{u}}$ and $\Delta Z_{j}:=Z_{j, \mathrm{~b}}-Z_{j, \mathrm{u}}$ :

$\left(1+\mathrm{Ka}_{T, \mathrm{~b}}\right) \Delta h=-\sum_{i=1}^{N_{\mathrm{S}}}\left(\frac{\mathrm{Ka}_{i, \mathrm{~b}}}{\mathrm{Le}_{i}}-\mathrm{Ka}_{T, \mathrm{~b}}\right) h_{i, \mathrm{ref}}\left(Y_{i, \mathrm{~b}}-Y_{i, \mathrm{u}}\right)$,
$\left(1+\mathrm{Ka}_{T, \mathrm{~b}}\right) \Delta Z_{j}=-\sum_{i=1}^{N_{\mathrm{s}}}\left(\frac{\mathrm{Ka}_{i, \mathrm{~b}}}{\mathrm{Le}_{i}}-\mathrm{Ka}_{T, \mathrm{~b}}\right) w_{j, i}\left(Y_{i, \mathrm{~b}}-Y_{i, \mathrm{u}}\right), \quad\left(j=1, \ldots, N_{\mathrm{e}}\right)$,

where the Karlovitz integrals $\mathrm{Ka}_{T, \mathrm{~b}}$ and $\mathrm{Ka}_{i, \mathrm{~b}}$ are defined by, respectively,

$\mathrm{Ka}_{T, \mathrm{~b}}:=\frac{1}{(\sigma m)_{\mathrm{b}}} \int_{s_{\mathrm{u}}}^{s_{\mathrm{b}}} \sigma(s) \rho(s) K(s) \tilde{T}(s) \mathrm{d} s$,

$\mathrm{Ka}_{i, \mathrm{~b}}:=\frac{\mathrm{Le}_{i}}{(\sigma m)_{\mathrm{b}}} \int_{s_{\mathrm{u}}}^{s_{\mathrm{b}}} \sigma(s) \rho(s) K(s) \tilde{Y}_{i}(s) \mathrm{d} s, \quad\left(i=1, \ldots, N_{\mathrm{s}}\right)$.

In (3.3) we introduced the normalized variables $\tilde{T}(s)$ and $\tilde{Y}_{i}(s)$, defined by

$\tilde{f}(s):=\frac{f(s)-f_{\mathrm{u}}}{f_{\mathrm{b}}-f_{\mathrm{u}}}, \quad\left(f=Y_{i}, T\right)$.

Clearly, $0=\tilde{f}_{\mathrm{u}} \leq \tilde{f}(s) \leq \tilde{f}_{\mathrm{b}}=1$ for $s_{\mathrm{u}} \leq s \leq s_{\mathrm{b}}$. Thus, $\mathrm{Ka}_{T, \mathrm{~b}}$ is the weighted mass-flow rate $\sigma(s) \rho(s) K(s) \tilde{T}(s)$ along the flame surfaces, integrated across the flame and scaled with the normal mass flow rate $(\sigma \mathrm{m})_{\mathrm{b}}$; a similar interpretation holds for $\mathrm{Ka}_{i, \mathrm{~b}}$.

Next, we will derive an expression for the mass burning rate $m_{\mathrm{b}}$. Consider the flamelet equation (2.6c) for the temperature, which can be written in the symbolic form

$A-\frac{\partial C}{\partial s}=S$

with $A, C$ and $S$ the advection, conduction and chemical source terms, respectively, defined by

$A:=\frac{\partial}{\partial s}(\sigma m T)+\sigma \rho K T, \quad C:=\sigma \frac{\lambda}{c_{p}} \frac{\partial T}{\partial s}, \quad S:=\sigma \omega_{T}$,

where we have assumed that $c_{p}=$ Const. The first term in $A$ describes advection in the $s$-direction and the second term, i.e., the stretch term $\sigma \rho K T$, advection along the flame surfaces. To find the first integral of equation (3.5a) we 
multiply it by $C$. Integrating the resulting equation across the flame from $s=s_{\mathrm{u}}$ to $s=s_{\mathrm{b}}$ and taking into account that the conduction fluxes vanish there, we obtain

$\int_{s_{\mathrm{u}}}^{s_{\mathrm{b}}} A(s) C(s) \mathrm{d} s=\int_{s_{\mathrm{u}}}^{s_{\mathrm{b}}} S(s) C(s) \mathrm{d} s$.

Substituting the appropriate expressions from (3.5b) and taking $T$ as the integration variable, the second integral can be rewritten as

$\int_{s_{\mathrm{u}}}^{s_{\mathrm{b}}} S(s) C(s) \mathrm{d} s=\int_{T_{\mathrm{u}}}^{T_{\mathrm{b}}} \sigma^{2} \frac{\lambda}{c_{p}} \omega_{T} \mathrm{~d} T$.

In order to determine the first integral in (3.6), we approximate $C$ by integrating equation (3.5a) over the preheat zone and neglecting the source term $S$. This way we obtain

$\int_{s_{\mathrm{u}}}^{s_{\mathrm{b}}} A(s) C(s) \mathrm{d} s \approx \int_{s_{\mathrm{u}}}^{s_{\mathrm{b}}} A(s)\left[\int_{s_{\mathrm{u}}}^{s} A(v) \mathrm{d} v\right] \mathrm{d} s=\frac{1}{2}\left[\int_{s_{\mathrm{u}}}^{s_{\mathrm{b}}} A(s) \mathrm{d} s\right]^{2}$.

This approximation of $C$ is justified since the reaction zone is usually much thinner than the preheat zone, so that the major contribution to the integral of $A(s) C(s)$ in (3.6) comes from the preheat zone. In fact, relation (3.8) is exact when the thickness of the reaction zone goes to zero for infinite activation energy, which we therefore assume in the following. Our approach is then equivalent to large-activation-energy asymptotics to determine the mass burning rate; see [22]. Next, substituting the expression for $A$ in (3.5b) and using the relation (3.1a) we find

$\int_{s_{\mathrm{u}}}^{s_{\mathrm{b}}} A(s) \mathrm{d} s=\left(1+\mathrm{Ka}_{T, \mathrm{~b}}\right)(\sigma m)_{\mathrm{b}}\left(T_{\mathrm{b}}-T_{\mathrm{u}}\right)$,

with the Karlovitz integral $\mathrm{Ka}_{T, \mathrm{~b}}$ defined in (3.3a). Combining (3.6-3.9) we obtain the following expression for the mass burning rate $m_{\mathrm{b}}$ in the burned gas mixture

$\left(1+\mathrm{Ka}_{T, \mathrm{~b}}\right)(\sigma m)_{\mathrm{b}}=F\left(h_{\mathrm{b}}, Z_{q, \mathrm{~b}}\right)$

where the function $F\left(h_{\mathrm{b}}, Z_{q, \mathrm{~b}}\right)$ is defined by

$F\left(h_{\mathrm{b}}, Z_{q, \mathrm{~b}}\right):=\frac{1}{T_{\mathrm{b}}-T_{\mathrm{u}}} \sqrt{2 \int_{T_{\mathrm{u}}}^{T_{\mathrm{b}}} \sigma^{2} \frac{\lambda}{c_{p}} \omega_{T} \mathrm{~d} T}$.

In $[12,21]$ we have explained that $F$ should be considered a function of the state vector $\psi_{\mathrm{b}}=\left(h_{\mathrm{b}}, Z_{j, \mathrm{~b}}\right)$ in the burned gas mixture. Note that for the special case of a stretchless flame with infinitely thin reaction zone we have $\left(\sigma m^{0}\right)_{\mathrm{b}}=F\left(h_{\mathrm{b}}^{0}, Z_{j, \mathrm{~b}}^{0}\right)$.

Next, we compare the mass burning rates for a stretched and a stretchless flame. For a given field $\sigma=\sigma(s)$, applying relation (3.10) for $K \neq 0$ and $K=0$, respectively, we can derive the following expression

$\left(1+\mathrm{Ka}_{T, \mathrm{~b}}\right) m_{\mathrm{b}}\left(\boldsymbol{\psi}_{\mathrm{b}}\right)=m_{\mathrm{b}}^{0}\left(\boldsymbol{\psi}_{\mathrm{b}}\right)$

where we have explicitly denoted the dependence of $m_{\mathrm{b}}$ and $m_{\mathrm{b}}^{0}$ on the vector $\boldsymbol{\psi}_{\mathrm{b}}$. Note that the variable $m_{\mathrm{b}}^{0}\left(\boldsymbol{\psi}_{\mathrm{b}}\right)$ in the right-hand side of (3.12) is the mass burning rate of a stretchless flame, which must be evaluated as a function of the state vector $\psi_{\mathrm{b}}$ of a stretched flame. In the derivation of (3.12) we have assumed, first, that the reaction zone is infinitely thin and, second, that the conductive flux vanishes at the burned and unburned sides of the flame. There are 
no other assumptions introduced and the relations (3.2), (3.3) and (3.12) together describe the effect of flame stretch and preferential diffusion on the mass burning rate of premixed flames. There is in principle no restriction on the stretch rate, curvature or reaction mechanism. It has been shown in a number of recent publications that this model accurately describes these influences for strongly stretched turbulent flames, modeled using DNS including reduced chemistry in the form of the flamelet-generated manifold technique and unit Lewis numbers [17,23]. Furthermore, it was recently observed that the model also successfully predicts stretch-related phenomena in weakly stretched methane and propane flames, modelled using complex chemistry in combination with non-unit Lewis numbers [18].

In the following we restrict ourselves to weak stretch because existing theories based on LAEA are restricted to weak stretch. Then, taking into account that $m_{\mathrm{b}}^{0}=m_{\mathrm{b}}^{0}\left(\boldsymbol{\psi}_{\mathrm{b}}^{0}\right), h_{\mathrm{b}}=h_{\mathrm{b}}^{0}+\Delta h$ and $Z_{j, \mathrm{~b}}=Z_{j, \mathrm{~b}}^{0}+\Delta Z_{j}\left(j=1, \ldots, N_{\mathrm{e}}\right)$, we can expand $m_{\mathrm{b}}^{0}\left(\psi_{\mathrm{b}}\right)$ in a Taylor series, to obtain

$\frac{m_{\mathrm{b}}-m_{\mathrm{b}}^{0}}{m_{\mathrm{b}}^{0}}=-\mathrm{Ka}_{T, \mathrm{~b}}+\Delta h \frac{\partial}{\partial h_{\mathrm{b}}}\left(\log m_{\mathrm{b}}^{0}\right)+\sum_{j=1}^{N_{\mathrm{e}}} \Delta Z_{j} \frac{\partial}{\partial Z_{j, \mathrm{~b}}}\left(\log m_{\mathrm{b}}^{0}\right)+$ h.o.t.

Expression (3.13) is only applicable to weakly stretched flames, i.e., $K$ is small enough to neglect second- (and higher-) order terms in the Karlovitz integrals; see (3.2) and (3.3). Otherwise, we can use both expressions (3.12) and (3.13) for arbitrary flames with multiple-species chemistry and transport. Thus, we do not have to impose any restrictions on the Lewis numbers, unlike in asymptotic theories, where $\left|\mathrm{Le}_{i}-1\right|$ are assumed to be small (of the order of the reciprocal Zeldovich number).

Summarizing, the derivation of an expression for the mass burning rate requires the following steps. First, we have to solve the flamelet equations (2.6) for the normalized combustion variables $\tilde{Y}_{i}(s)$ and $\tilde{T}(s)$, either analytically or numerically. In the following sections, we will use analytical expressions for $\tilde{Y}_{i}^{0}(s)$ and $\tilde{T}^{0}(s)$, assuming constant coefficients. Second, we have to compute the Karlovitz integrals in (3.3). With regard to the density in these integrals, we distinguish two case, i.e., constant density and variable density. Third, we determine from (3.2) the differences $\Delta h$ and $\Delta Z_{j}$ due to differential diffusion and flame stretch. Finally, assuming that $K$ is small enough to neglect higher-order terms, we have to evaluate expression (3.13).

\section{Mass burning rate: the constant-density approximation}

We will elaborate the formulae (3.2), (3.3) and (3.13) for the special case of a lean stagnation flame in a constantdensity flow, thus ignoring expansion of the gas mixture due to combustion. So, consider a model flame determined by a one-step irreversible reaction $\mathcal{F} \rightarrow \mathcal{P}$, with one rate-determining lean species $\mathcal{F}$. The main assumptions are:

I The flame is flat, i.e., $\sigma(s)=1$.

II Both species have the same Lewis number, i.e., $\operatorname{Le}_{\mathcal{F}}=\operatorname{Le}_{\mathcal{P}}=\mathrm{Le}$.

III The thermal conductivity $\lambda$ and the specific heat $c_{p}$ are constant.

IV The flame is weakly stretched.

$\mathrm{V}$ The density is constant, i.e., $\rho(s)=$ Const $=\rho_{\mathrm{b}}^{0}$.

VI The reaction zone is infinitely thin and located at $s=s_{\mathrm{b}}=0$ and the preheat zone extends to infinity, i.e., $s_{\mathrm{u}} \rightarrow-\infty$.

As a consequence of assumption IV, we can neglect the higher-order terms in (3.13) and replace the combustion variables in (3.2) and (3.3) by their stretchless counterparts. Moreover, we can prove that in the preheat zone $K(s)$ is nearly constant and equal to the applied strain rate $a$; see the Appendix. Therefore, we take $K(s)=a$ in the preheat zone.

First, we solve the stretchless flamelet equations (2.6) for the combustion variables $\tilde{T}^{0}(s), \tilde{Y}_{\mathcal{F}}^{0}(s)$ and $\tilde{Y}_{\mathcal{P}}^{0}(s)$. Note that $m^{0}(s)=$ Const $=m_{\mathrm{u}}^{0}=m_{\mathrm{b}}^{0}=m^{0}$. Since the reaction zone is considered infinitely thin, we can replace the thermal/chemical source terms by delta functions, i.e., $\omega_{T}=Q \delta(s)$, where the coefficient $Q$ should be chosen 


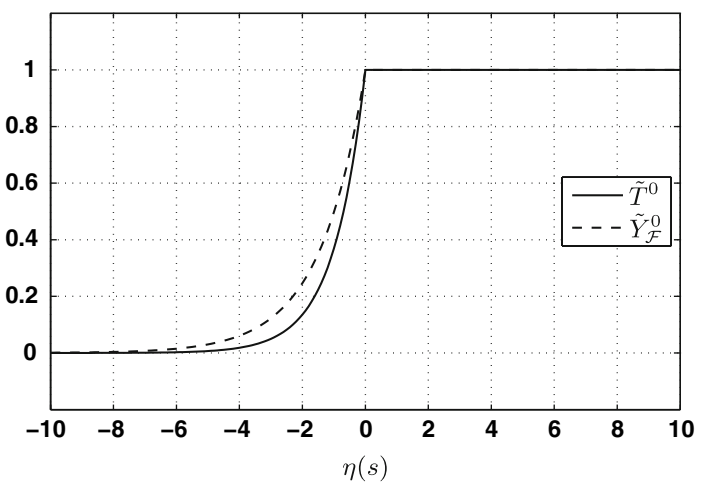

Fig. 1 The normalized variables $\tilde{T}^{0}(s)$ and $\tilde{Y}_{\mathcal{F}}^{0}(s)$, for Le $=0.7$, as a function of the dimensionless coordinate $\eta(s)$

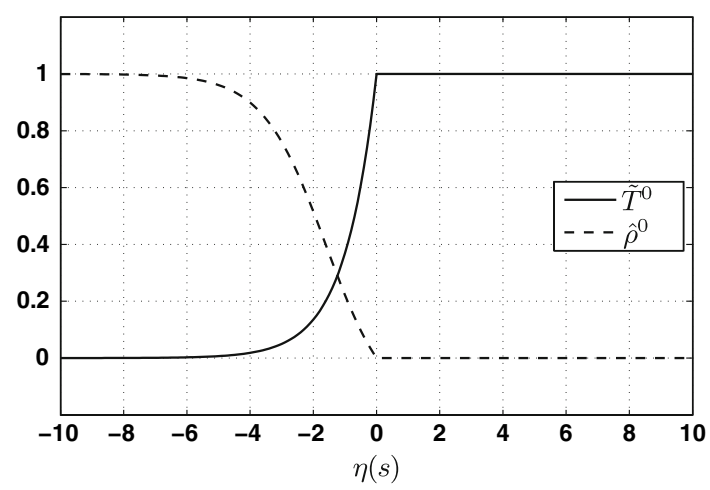

Fig. 2 The normalized variables $\tilde{T}^{0}(s)$ and $\hat{\rho}^{0}(s):=1-\tilde{\rho}^{0}(s)$, for $\theta=5$, as a function of the dimensionless coordinate $\eta(s)$

such that $T(s)$ remains bounded for $s \rightarrow \infty$, and likewise for $\omega_{i}$. This way, we obtain in the preheat zone $s<0$ the solution

$\tilde{T}^{0}(s)=\mathrm{e}^{\eta(s)}, \quad \tilde{Y}_{\mathcal{F}}^{0}(s)=\tilde{Y}_{\mathcal{P}}^{0}(s)=\mathrm{e}^{\mathrm{Le} \eta(s)}, \quad \eta(s):=\frac{s}{\delta_{\mathrm{f}}}, \quad \delta_{\mathrm{f}}:=\frac{\lambda}{m^{0} c_{p}}$,

where $\eta(s)$ is the scaled coordinate in the preheat zone and $\delta_{\mathrm{f}}$ is the thermal thickness of the stretchless flame. On the other hand, in the burned gas mixture, we have $\tilde{T}^{0}(s)=\tilde{Y}_{\mathcal{F}}^{0}(s)=\tilde{Y}_{\mathcal{P}}^{0}(s)=1$, with $s>0$ the usual Cartesian coordinate; see Fig. 1.

Substituting the stretchless solution (4.1) in (3.3), we obtain the following expressions for the Karlovitz integrals

$\mathrm{Ka}_{T, \mathrm{~b}}=\mathrm{Ka}_{\mathcal{F}, \mathrm{b}}=\mathrm{Ka}_{\mathcal{P}, \mathrm{b}}=\mathrm{Ka}_{\mathrm{b}}, \quad \mathrm{Ka}_{\mathrm{b}}:=\frac{a \delta_{\mathrm{f}} \rho_{\mathrm{b}}^{0}}{m^{0}}$

where $\mathrm{Ka}_{\mathrm{b}}$ is the usual Karlovitz number at the burned side of the flame.

Next, we have to compute the differences $\Delta h$ and $\Delta Z_{j}$. Since the one-step reaction $\mathcal{F} \rightarrow \mathcal{P}$ involves only one element, its mass fraction $Z$ has to be constant, i.e., $Z(s)=$ Const, and consequently all $\Delta Z_{j}$-terms in (3.13) cancel. Taking into account that $\mathcal{F}$ and $\mathcal{P}$ have the same Lewis number and Karlovitz integral, we can write the $\Delta h$-term as

$$
\begin{aligned}
\Delta h & =\left(h_{\mathcal{F}, \text { ref }} Y_{\mathcal{F}, \mathrm{u}}^{0}-h_{\mathcal{P}, \text { ref }} Y_{\mathcal{P}, \mathrm{b}}^{0}\right)\left(\frac{1}{\mathrm{Le}}-1\right) \mathrm{Ka}_{\mathrm{b}}+\mathcal{O}\left(\mathrm{Ka}_{\mathrm{b}}^{2}\right) \\
& =c_{p}\left(T_{\mathrm{b}}^{0}-T_{\mathrm{u}}^{0}\right)\left(\frac{1}{\mathrm{Le}}-1\right) \mathrm{Ka}_{\mathrm{b}}+\mathcal{O}\left(\mathrm{Ka}_{\mathrm{b}}^{2}\right) .
\end{aligned}
$$

The second equality in (4.3) follows readily from the definition of $h$ in (2.8) and the relation $\Delta h^{0}=0$. For the mass burning rate $m^{0}$ we adopt the same model as used by Barenblatt et al. [24], i.e.,

$m^{0}=m^{0}\left(T_{\mathrm{b}}\right)=$ Const $\mathrm{e}^{-T_{\mathrm{a}} /\left(2 T_{\mathrm{b}}\right)}$,

where $T_{\mathrm{a}}:=E_{\mathrm{a}} / R$ is the activation temperature and $E_{\mathrm{a}}$ the activation energy. Combining (3.13), (4.2)-(4.4) and applying the chain rule

$\frac{\partial}{\partial h_{\mathrm{b}}}\left(\log m^{0}\right)=\frac{1}{c_{p}} \frac{\partial}{\partial T_{\mathrm{b}}}\left(\log m^{0}\right)$,

we get the following relation 


$$
\begin{aligned}
\frac{m_{\mathrm{b}}-m^{0}}{m^{0}} & =-\mathrm{Ka}_{\mathrm{b}}+c_{p}\left(T_{\mathrm{b}}^{0}-T_{\mathrm{u}}^{0}\right)\left(\frac{1}{\mathrm{Le}}-1\right) \mathrm{Ka}_{\mathrm{b}} \frac{T_{\mathrm{a}}}{2\left(T_{\mathrm{b}}^{0}\right)^{2}} \frac{1}{c_{p}}+\mathcal{O}\left(\mathrm{Ka}_{\mathrm{b}}^{2}\right) \\
& =-\left(1+\frac{\mathrm{Ze}}{2}\left(1-\frac{1}{\mathrm{Le}}\right)\right) \mathrm{Ka}_{\mathrm{b}}+\mathcal{O}\left(\mathrm{Ka}_{\mathrm{b}}^{2}\right),
\end{aligned}
$$

where $\mathrm{Ze}:=T_{\mathrm{a}}\left(T_{\mathrm{b}}^{0}-T_{\mathrm{u}}^{0}\right) /\left(\left(T_{\mathrm{b}}^{0}\right)^{2}\right)$ is the Zeldovich number [25, p. 155]. Finally, we can rewrite relation (4.5) in the equivalent form

$$
\frac{m_{\mathrm{b}}-m^{0}}{m^{0}}=-\mathcal{M}_{\mathrm{b}} \mathrm{Ka}_{\mathrm{b}}+\mathcal{O}\left(\mathrm{Ka}_{\mathrm{b}}^{2}\right)
$$

where $\mathcal{M}_{\mathrm{b}}$ is the Markstein number given by

$$
\mathcal{M}_{\mathrm{b}}=1+\frac{\mathrm{Ze}}{2}\left(1-\frac{1}{\mathrm{Le}}\right)
$$

The Markstein number is a measure of the relative change in the mass burning rate $m_{\mathrm{b}}$ due to flame stretch. The second term in $\mathcal{M}_{\mathrm{b}}$ accounts for preferential diffusion when Le $\neq 1$.

\section{Mass burning rate: variable density}

In a similar way as in Sect. 4, we derive an expression for the mass burning rate $m_{\mathrm{b}}$, but now for variable density, thus accounting for gas expansion due to combustion. All assumptions remain valid, except assumption V. Instead, we determine the density from the equation of state (2.7). Consequently, we have the following expression for the density in the preheat zone

$\rho^{0}(s)=\frac{\rho_{\mathrm{b}}^{0} T_{\mathrm{b}}^{0}}{T^{0}(s)}=\rho_{\mathrm{b}}^{0} \frac{\theta+1}{1+\theta \tilde{T}^{0}(s)}, \quad \theta:=\frac{T_{\mathrm{b}}^{0}-T_{\mathrm{u}}^{0}}{T_{\mathrm{u}}^{0}}=\frac{\rho_{\mathrm{u}}^{0}-\rho_{\mathrm{b}}^{0}}{\rho_{\mathrm{b}}^{0}} ;$

see Fig. 2. In the derivation of (5.1) we have used that $\rho^{0}(s) T^{0}(s)=$ Const $=\rho_{\mathrm{b}}^{0} T_{\mathrm{b}}^{0}$, which is a consequence of the equation of state (2.7) and the assumption that $W=$ Const. The parameter $\theta$ is the thermal-expansion coefficient. Obviously, in the burned gas mixture $\rho(s)=\rho_{\mathrm{b}}^{0}$. Thus, we can interpret the preheat zone as a layer of variable density connecting the incompressible unburned and burned gas mixtures. The expressions in (4.1) for the combustion variables in the preheat zone still hold.

Substituting the formulae for $\rho^{0}(s)$ and $\tilde{T}^{0}(s)$ in (3.3a), we obtain the following expression for the Karlovitz integral $\mathrm{Ka}_{T, \mathrm{~b}}$

$\mathrm{Ka}_{T, \mathrm{~b}}=\mathrm{Ka}_{\mathrm{b}}(\theta+1) \frac{\log (\theta+1)}{\theta}$.

Likewise, substituting the expressions for $\rho^{0}(s), \tilde{T}^{0}(s)$ and $\tilde{Y}_{\mathcal{F}}^{0}(s)$ in $(3.3 \mathrm{~b})$, we obtain for $\mathrm{Ka} \mathcal{F}, \mathrm{b}$ the expression

$$
\frac{\mathrm{Ka} \mathcal{F}, \mathrm{b}}{\mathrm{Le}}=\mathrm{Ka}_{\mathrm{b}}(\theta+1) \int_{0}^{1} \frac{v^{\mathrm{Le}-1}}{1+\theta v} \mathrm{~d} \nu .
$$

The integral in (5.3) can be expressed in terms of hypergeometric functions; see e.g. [26, Sect. 15.3, formula 15.3.1]. Note that, in the limit for $\theta \rightarrow 0$, the expressions for $\mathrm{Ka}_{T, \mathrm{~b}}$ and $\mathrm{Ka}_{\mathcal{F}, \mathrm{b}}$ reduce to the ones in (4.2). 
Next, we have to compute the enthalpy difference $\Delta h$. Analogous to the derivation in the previous section, we find

$\Delta h=c_{p}\left(T_{\mathrm{b}}^{0}-T_{\mathrm{u}}^{0}\right)\left(\frac{\mathrm{Ka} \mathcal{F}, \mathrm{b}}{\mathrm{Le}}-\mathrm{Ka}_{T, \mathrm{~b}}\right)+\mathcal{O}\left(\mathrm{Ka}_{\mathrm{b}}^{2}\right)$,

with $\mathrm{Ka}_{T, \mathrm{~b}}$ and $\mathrm{Ka}_{\mathcal{F}, \mathrm{b}}$ given in (5.2) and (5.3), respectively. Using relation (4.4) for the mass burning rate $m^{0}$, we get the following generalization of (4.5),

$\frac{m_{\mathrm{b}}-m^{0}}{m^{0}}=-\mathrm{Ka}_{T, \mathrm{~b}}+\frac{\mathrm{Ze}}{2}\left(\frac{\mathrm{Ka}_{\mathcal{F}, \mathrm{b}}}{\mathrm{Le}}-\mathrm{Ka}_{T, \mathrm{~b}}\right)+\mathcal{O}\left(\mathrm{Ka}_{\mathrm{b}}^{2}\right)$.

Applying integration by parts to the integral in (5.3) and subsequently substituting the resulting expression for $\mathrm{Ka}_{\mathcal{F}, \mathrm{b}} / \mathrm{Le}-\mathrm{Ka}_{T, \mathrm{~b}}$ in (5.5), we obtain the familiar relation (4.6), with the Markstein number $\mathcal{M}_{\mathrm{b}}$ given by

$\mathcal{M}_{\mathrm{b}}=(\theta+1) \frac{\log (\theta+1)}{\theta}+\frac{\mathrm{Ze}}{2}(\mathrm{Le}-1) \frac{\theta+1}{\theta} \int_{0}^{1} v^{\mathrm{Le}-2} \log (1+\theta v) \mathrm{d} v$

The first term in $\mathcal{M}_{\mathrm{b}}$ describes the effect of gas expansion on the mass burning rate $m_{\mathrm{b}}$ and the second term the effect of preferential diffusion. Note that for $\theta \rightarrow 0$ expression (5.6) reduces to (4.7).

\section{The mass burning rate in the unburned mixture}

In the previous sections we derived expressions for the mass burning rate $m_{\mathrm{b}}$ at the burned side of the flame, i.e., at the reaction layer. Alternatively, in this section we derive a model for the mass burning rate $m_{\mathrm{u}}$ in the unburned gas mixture, equivalent to the model for $m_{\mathrm{b}}$ presented in Sect. 3. First, we give alternative expressions for $\Delta h$ and $\Delta Z_{j}$. Analogous to the derivation of (3.2), we find

$$
\begin{aligned}
& \left(1+\mathrm{Ka}_{T, \mathrm{u}}\right) \Delta h=-\sum_{i=1}^{N_{\mathrm{s}}}\left(\frac{\mathrm{Ka}_{i, \mathrm{u}}}{\mathrm{Le}_{i}}-\mathrm{Ka}_{T, \mathrm{u}}\right) h_{i, \mathrm{ref}}\left(Y_{i, \mathrm{~b}}-Y_{i, \mathrm{u}}\right), \\
& \left(1+\mathrm{Ka}_{T, \mathrm{u}}\right) \Delta Z_{j}=-\sum_{i=1}^{N_{\mathrm{s}}}\left(\frac{\mathrm{Ka}_{i, \mathrm{u}}}{\mathrm{Le}_{i}}-\mathrm{Ka}_{T, \mathrm{u}}\right) w_{j, i}\left(Y_{i, \mathrm{~b}}-Y_{i, \mathrm{u}}\right), \quad\left(j=1, \ldots, N_{\mathrm{e}}\right),
\end{aligned}
$$

where the Karlovitz integrals $\mathrm{Ka}_{T, \mathrm{u}}$ and $\mathrm{Ka}_{i, \mathrm{u}}$ are defined by, respectively,

$$
\begin{aligned}
\mathrm{Ka}_{T, \mathrm{u}} & :=\frac{-1}{(\sigma m)_{\mathrm{u}}} \int_{s_{\mathrm{u}}}^{s_{\mathrm{b}}} \sigma(s) \rho(s) K(s)(1-\tilde{T}(s)) \mathrm{d} s, \\
\mathrm{Ka}_{i, \mathrm{u}} & :=\frac{-\mathrm{Le}_{i}}{(\sigma m)_{\mathrm{u}}} \int_{s_{\mathrm{u}}}^{s_{\mathrm{b}}} \sigma(s) \rho(s) K(s)\left(1-\tilde{Y}_{i}(s)\right) \mathrm{d} s, \quad\left(i=1, \ldots, N_{\mathrm{s}}\right),
\end{aligned}
$$

i.e., the Karlovitz integral $\mathrm{Ka}_{T, \mathrm{u}}$ is the weighted mass-flow rate $\sigma(s) \rho(s) K(s)(1-\tilde{T}(s))$, integrated across the flame and scaled with the normal mass-flow rate $-(\sigma m)_{\mathrm{u}}$ and likewise for $\mathrm{Ka}_{i, \mathrm{u}}$. From their definitions and the flamelet equation (2.6a), we can derive the following relations between the Karlovitz integrals $\mathrm{Ka}_{T, \mathrm{~b}}, \mathrm{Ka}_{i, \mathrm{~b}}$ for the burned side of the flame, and $\mathrm{Ka}_{T, \mathrm{u}}, \mathrm{Ka}_{i, \mathrm{u}}$ for the unburned side, i.e.,

$$
\begin{aligned}
& (\sigma m)_{\mathrm{b}}\left(\mathrm{Ka}_{T, \mathrm{~b}}+1\right)=(\sigma m)_{\mathrm{u}}\left(\mathrm{Ka}_{T, \mathrm{u}}+1\right), \\
& (\sigma m)_{\mathrm{b}}\left(\mathrm{Ka}_{i, \mathrm{~b}}+\mathrm{Le}_{i}\right)=(\sigma m)_{\mathrm{u}}\left(\mathrm{Ka}_{i, \mathrm{u}}+\mathrm{Le}_{i}\right) .
\end{aligned}
$$


Next, equivalent to relation (3.12), we have the following relation for $m_{\mathrm{u}}$,

$$
\left(1+\mathrm{Ka}_{T, \mathrm{u}}\right) m_{\mathrm{u}}\left(\boldsymbol{\psi}_{\mathrm{b}}\right)=m_{\mathrm{u}}^{0}\left(\boldsymbol{\psi}_{\mathrm{b}}\right)
$$

where we again assume that $m_{\mathrm{u}}^{0}$ depends on the conserved variables $\psi_{\mathrm{b}}$ in the burned gas mixture. For weakly stretched flames, we can expand $m_{\mathrm{u}}^{0}\left(\boldsymbol{\psi}_{\mathrm{b}}\right)$ in a Taylor series, to find

$\frac{m_{\mathrm{u}}-m_{\mathrm{u}}^{0}}{m_{\mathrm{u}}^{0}}=-\mathrm{Ka}_{T, \mathrm{u}}+\Delta h \frac{\partial}{\partial h_{\mathrm{b}}}\left(\log m_{\mathrm{u}}^{0}\right)+\sum_{j=1}^{N_{\mathrm{e}}} \Delta Z_{j} \frac{\partial}{\partial Z_{j, \mathrm{~b}}}\left(\log m_{\mathrm{u}}^{0}\right)+$ h.o.t.

Summarizing, the model for $m_{\mathrm{u}}$ consists of either relation (6.4) or (6.5), coupled with (6.1) and (6.2) to determine the differences $\Delta h$ and $\Delta Z_{j}$.

In the following, we restrict ourselves to the variable-density case of the stagnation flame of the previous sections, and use the stretchless variables in (4.1) to derive expressions for the Karlovitz integrals and for the difference $\Delta h$. Straightforward substitution of (4.1) and (5.1) in (6.2) would lead to divergent Karlovitz integrals for $s_{\mathrm{u}} \rightarrow-\infty$. The reason for this divergence is that $(1-\tilde{T}(s)) K(s) \neq 0$ in the entire flame and does not tend to 0 for $s \rightarrow-\infty$. Consequently, the mass burning rate $m_{\mathrm{u}}$ becomes unbounded for $s_{\mathrm{u}} \rightarrow-\infty$, in agreement with the flamelet equation (2.6a). To remedy this problem, we cut off the integration interval at some finite reference value $s_{\text {ref }}$. Then, substituting $v=\exp \left(s / \delta_{\mathrm{f}}\right)$ and applying the method of partial fractions, we obtain

$$
\begin{aligned}
& \mathrm{Ka}_{T, \mathrm{u}} \sim \mathrm{Ka}_{\mathrm{u}}\left(\eta_{\text {ref }}+(\theta+1) \frac{\log (\theta+1)}{\theta}\right), \quad \eta_{\text {ref }}:=\frac{s_{\text {ref }}}{\delta_{\mathrm{f}}}, \quad \mathrm{Ka}_{\mathrm{u}}:=\frac{a \delta_{\mathrm{f}} \rho_{\mathrm{u}}^{0}}{m^{0}}, \\
& \frac{\mathrm{Ka}_{\mathcal{F}, \mathrm{u}}}{\mathrm{Le}} \sim \mathrm{Ka}_{\mathrm{u}}\left(\eta_{\text {ref }}+\log (\theta+1)+\int_{0}^{1} \frac{v^{\mathrm{Le}-1}}{1+\theta v} \mathrm{~d} \nu\right),
\end{aligned}
$$

where $\mathrm{Ka}_{\mathrm{u}}$ is the Karlovitz number in the unburned gas mixture. Note that $\mathrm{Ka}_{\mathrm{u}}=(\theta+1) \mathrm{Ka}_{\mathrm{b}}$. Next, we have to derive an expression for $\Delta h$ from (6.1a). Applying the same assumptions as before, we find

$$
\Delta h=c_{p}\left(T_{\mathrm{b}}^{0}-T_{\mathrm{u}}^{0}\right)\left(\frac{\mathrm{Ka}_{\mathcal{F}, \mathrm{u}}}{\mathrm{Le}}-\mathrm{Ka}_{T, \mathrm{u}}\right)+\mathcal{O}\left(\mathrm{Ka}_{\mathrm{u}}^{2}\right) .
$$

Note from relations (6.6), that the Karlovitz integrals diverge, whereas $\Delta h$ remains bounded for $\eta_{\text {ref }} \rightarrow-\infty$. Substituting (6.7) in relation (6.5), we find for the relative change in $m_{\mathrm{u}}$ the following expression

$$
\frac{m_{\mathrm{u}}-m^{0}}{m^{0}}=-\mathrm{Ka}_{T, \mathrm{u}}+\frac{\mathrm{Ze}}{2}\left(\frac{\mathrm{Ka}_{\mathcal{F}, \mathrm{u}}}{\mathrm{Le}}-\mathrm{Ka}_{T, \mathrm{u}}\right)+\mathcal{O}\left(\mathrm{Ka}_{\mathrm{u}}^{2}\right) .
$$

Finally, substituting the expressions for the Karlovitz integrals in (6.6) and applying integration by parts to the integral involved, we can rewrite relation (6.8) in the form

$$
\frac{m_{\mathrm{u}}-m^{0}}{m^{0}}=-\mathcal{M}_{\mathrm{u}}\left(\eta_{\mathrm{ref}}\right) \mathrm{Ka}_{\mathrm{u}}+\mathcal{O}\left(\mathrm{Ka}_{\mathrm{u}}^{2}\right),
$$

where the Markstein number at the unburned side of the flame is given by

$$
\begin{aligned}
\mathcal{M}_{\mathrm{u}}\left(\eta_{\text {ref }}\right) & =\eta_{\text {ref }}+(\theta+1) \frac{\log (\theta+1)}{\theta}+\frac{\mathrm{Ze}}{2}(\mathrm{Le}-1) \frac{1}{\theta} \int_{0}^{1} v^{\mathrm{Le}-2} \log (1+\theta v) \mathrm{d} \nu \\
& =\eta_{\text {ref }}+\frac{1}{\theta+1} \mathcal{M}_{\mathrm{b}}+\log (\theta+1) .
\end{aligned}
$$

Thus, expressions (4.6) and (5.6) for $m_{\mathrm{b}}$ and $\mathcal{M}_{\mathrm{b}}$ can be used to determine the influence of flame stretch on the mass burning rate in the burned gases at the reaction layer $\eta(s)=0$. On the other hand, expressions (6.9) and (6.10) 
for $m_{\mathrm{u}}$ and $\mathcal{M}_{\mathrm{u}}$ describe what happens in the unburned gases, but as there is no natural position in the unburned gases, the reference position $\eta=\eta_{\text {ref }}$ indicates the point where $m_{\mathrm{u}}$ is considered. Thus, compared to expression (5.6) for $\mathcal{M}_{\mathrm{b}}$, this expression contains the additional term $\eta_{\text {ref }}$, that accounts for the finite thickness of the preheat zone. Note that the above relation implies that there is a difference between $m_{\mathrm{u}}$ and $m_{\mathrm{b}}$, given by

$\frac{m_{\mathrm{u}}-m_{\mathrm{b}}}{m^{0}}=\left(-\eta_{\mathrm{ref}}-\log (\theta+1)\right) \mathrm{Ka}_{\mathrm{u}}$,

which is not equal to zero-even if they are evaluated at the same position, i.e., if we choose $\eta_{\text {ref }}=0$. The term proportional to $\log (\theta+1)$ is related to the gas expansion in the preheat zone. In the limit of no expansion, i.e., $\theta \rightarrow 0$, relation $(6.10)$ reduces to

$\mathcal{M}_{\mathrm{u}}\left(\eta_{\mathrm{ref}}\right)=\eta_{\mathrm{ref}}+1+\frac{\mathrm{Ze}}{2}\left(1-\frac{1}{\mathrm{Le}}\right)=\mathcal{M}_{\mathrm{b}}+\eta_{\text {ref }}$

which gives identical Markstein numbers and mass burning rates at $\eta_{\mathrm{ref}}=0$.

\section{A review on the mass burning rate}

In this final section we review the relations for the mass burning rate derived in the previous sections. In particular, we compare it to results obtained with LAEA from literature and show the consistency of our model. Finally, we will discuss the implications for numerical and experimental studies.

In order to compare our results with literature, we first have to relate our stretch rate $K$ with the classical stretch rate $\kappa$, which is used in flame sheet models, where flames are considered as a single surface separating the burned and unburned gases. It is defined as the fractional rate of change of the area element on the flame sheet when it is moving with velocity $\boldsymbol{v}_{\mathrm{f}}$. For a properly defined coordinate system $\boldsymbol{\xi}=\boldsymbol{\xi}(\boldsymbol{x}, t)$ for which the scale factor $h_{1}=$ Const, we have

$K=\kappa+\frac{1}{\rho} \frac{\partial \rho}{\partial \tau}, \quad \kappa:=\frac{1}{\sigma} \frac{\partial \sigma}{\partial \tau}$,

with $\sigma$ defined in (2.5). Since by definition $\partial T / \partial \tau=0$ and $\rho T=$ Const by virtue of the equation of state (2.7), we also have $\partial \rho / \partial \tau=0$, and consequently $K=\kappa$. We like to emphasize that this equality only holds at the flame sheet, since $\kappa$ is not defined elsewhere. Next, we will reformulate our results in terms of the laminar burning velocity $s_{\mathrm{L}}$. Assuming weak stretch, so that we can replace $\rho_{\mathrm{b}}$ by $\rho_{\mathrm{b}}^{0}$, we obtain at the burned side of the flame

$\frac{s_{\mathrm{L}, \mathrm{b}}-s_{\mathrm{L}, \mathrm{b}}^{0}}{s_{\mathrm{L}, \mathrm{b}}^{0}}=-\mathcal{M}_{\mathrm{b}} \mathrm{Ka}_{\mathrm{b}}+\mathcal{O}\left(\mathrm{Ka}_{\mathrm{b}}^{2}\right), \quad \mathrm{Ka}_{\mathrm{b}}:=\frac{\kappa \delta_{\mathrm{f}}}{s_{\mathrm{L}, \mathrm{b}}^{0}}$,

with the Markstein number $\mathcal{M}_{\mathrm{b}}$ defined in (4.7) for the constant density approximation and in (5.6) for variable density. Remember that the superscript 0 refers to the value of the corresponding variable for a stretchless flame. Relation (7.2) was previously derived by Joulin and Clavin for the constant density case and by Clavin for the variable density case. However, their Markstein numbers differ slightly from ours. In particular, according to Joulin and Clavin [16]

$\mathcal{M}_{\mathrm{b}}=1+\frac{\mathrm{Ze}}{2}(\mathrm{Le}-1)$ 
whereas Clavin obtained the result [7]

$\mathcal{M}_{\mathrm{b}}=(\theta+1) \frac{\log (\theta+1)}{\theta}+\frac{\mathrm{Ze}}{2}(\mathrm{Le}-1) \frac{\theta+1}{\theta} \int_{0}^{1} v^{-1} \log (1+\theta v) \mathrm{d} \nu$.

In their derivations, they considered the flame as a surface separating the (incompressible) burned and unburned gas mixtures. They first integrated the conservation equations across the reaction zone, to give boundary conditions and jump conditions for the flow in the (un)burned gas mixtures, which they subsequently solved using asymptotic expansions. However, in their derivations they had to assume that $|\mathrm{Le}-1|=\mathcal{O}(1 / \mathrm{Ze})$, so that (7.3a) and (7.3b) are to lowest order in $1 /$ Ze equal to (4.7) and (5.6), respectively. Our expressions do not suffer from this assumption and are valid for general Lewis numbers.

Alternatively, at the unburned side of the flame we have

$\frac{s_{\mathrm{L}, \mathrm{u}}-s_{\mathrm{L}, \mathrm{u}}^{0}}{s_{\mathrm{L}, \mathrm{u}}^{0}}=-\mathcal{M}_{\mathrm{u}}\left(\eta_{\mathrm{ref}}\right) \mathrm{Ka}_{\mathrm{u}}+\mathcal{O}\left(\mathrm{Ka}_{\mathrm{u}}^{2}\right), \quad \mathrm{Ka}_{\mathrm{u}}:=\frac{\kappa \delta_{\mathrm{f}}}{s_{\mathrm{L}, \mathrm{u}}^{0}}$,

with the Markstein number $\mathcal{M}_{\mathrm{u}}\left(\eta_{\text {ref }}\right)$ given in (6.10). A very similar result was obtained by Clavin and Williams [7]; they found (7.4), with the Markstein number $\mathcal{M}_{\mathrm{u}}$ given by

$\mathcal{M}_{\mathrm{u}}=(\theta+1) \frac{\log (\theta+1)}{\theta}+\frac{\mathrm{Ze}}{2}(\mathrm{Le}-1) \frac{1}{\theta} \int_{0}^{1} v^{-1} \log (1+\theta v) \mathrm{d} \nu$.

Apart from the small difference in the preferential diffusion term, again due to the fact that Clavin and Williams had to assume $|\mathrm{Le}-1|=\mathcal{O}(1 / \mathrm{Ze})$, the term $\eta_{\text {ref }}$ is also missing. This is a consequence of their model, where the entire flame is considered a surface, i.e., they implicitly take $\eta_{\text {ref }}=0$.

This shows indeed that the LAEA theory uses the position $s=0$ (at the reaction layer) to identify the unburned mass burning rate $m_{\mathrm{u}}$ as well as the burned mass burning rate $m_{\mathrm{b}}$. This is a direct consequence of the LAEA theory which assumes that the complete flame structure is viewed as an interface (at $s=0$ ) separating unburned and burned gases, in contrast with the IA where the flame has a finite thickness. What the LAEA theory implicitly comes down to is that the asymptotic behavior of $m(s)$ in the (un)burned gases is evaluated and that it is extrapolated linearly to the position $s=0$ to identify the reported results for $m_{\mathrm{b}}$ and $m_{\mathrm{u}}$. Or, alternatively, the limit of an infinitely thin flame is taken, i.e., $\delta_{\mathrm{f}} \rightarrow 0$ (reaction zone including preheat zone). This procedure gives different results for $m_{\mathrm{b}}$ and $m_{\mathrm{u}}$ (or $\mathcal{M}_{\mathrm{b}}$ and $\mathcal{M}_{\mathrm{u}}$ ), although they are evaluated at the same point.

To understand this difference more clearly, let us now study the variation of $m(s)$ in the flame. We will consider two cases: as reference we will consider the theoretical model considered in this paper where the preheat zone thickness is infinite, while the reaction zone is infinitely thin (see Fig. 3) and compare this with a numerical simulation where both the reaction zone and the preheat zone have a finite thickness (see Fig. 4). We can determine the mass burning rate $m(s)$ from the first flamelet equation in (2.6), that describes conservation of mass. Thus, integrating Eq. (2.6a) from $s=s_{\mathrm{b}}=0$, where the infinitely thin reaction zone is situated, to a location $s \neq 0$ in either the preheat zone or the burned gas mixture, we obtain

$m(s)-m_{\mathrm{b}}=-a \int_{0}^{s} \rho^{0}\left(s^{\prime}\right) \mathrm{d} s^{\prime}$

where we have replaced $\rho$ by its stretchless counterpart $\rho^{0}$. Note that we have taken $K(s)=a$, which is justified for the complete preheat zone and the burned gas mixture in the vicinity of the reaction layer; see Appendix. In the burned gas mixture, for $s>0$, we may take $\rho^{0}(s)=\rho_{\mathrm{b}}^{0}$, to find the mass burning rate linearly decreasing with $\eta(s)$, i.e., 


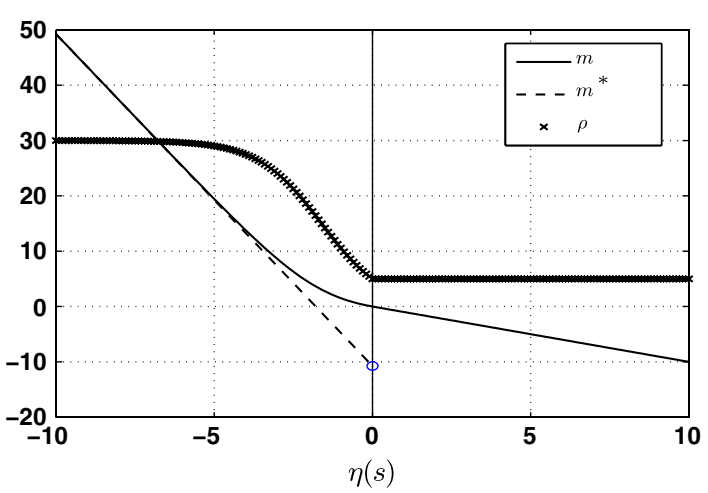

Fig. 3 The (scaled) mass burning rate $m(s)$, its linear approximation $m^{*}(s)$ and density $\rho(s)$ of a flame with infinitely thin reaction layer at $\eta(s)=0$ subject to a constant stretch rate $K(s)=a$

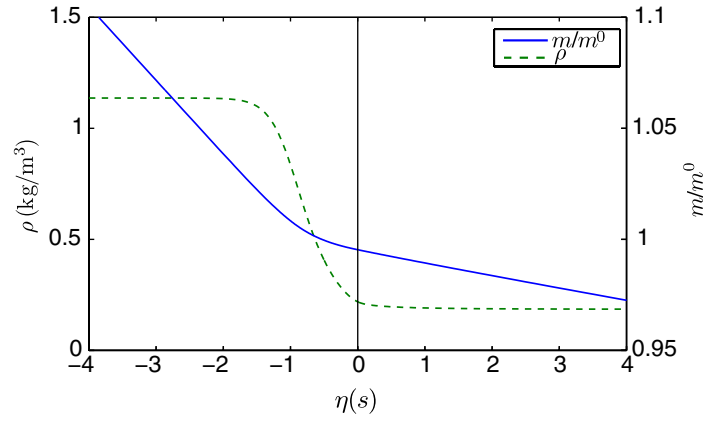

Fig. 4 The mass burning rate $m(s)$ and the density $\rho(s)$ of a methane/air flame computed numerically from the flamelet equations (2.6) using GRI-mech 3.0. Parameter values are: $\varphi=0.7, a=10 \mathrm{~s}^{-1}, \delta_{\mathrm{f}}=6.22 \times 10^{-2} \mathrm{~cm}, m^{0}=2.13 \times$ $10^{-2} \mathrm{~g} / \mathrm{cm}^{2} \mathrm{~s}$

$\frac{m(s)-m_{\mathrm{b}}}{m^{0}}=-\mathrm{Ka}_{\mathrm{b}} \eta(s)$,

which has been plotted in Fig. 3 (at the right where $\eta(s)>0$ ). This linear decrease of $m(s)$ in the burned gases can also be seen in Fig. 4, which presents numerical results for a weakly strained lean methane-air flame (equivalence ratio $\varphi=0.7$, strain rate $a=10 \mathrm{~s}^{-1}$ ) computed from the full set of conservation equations using GRI-mech 3.0. Let us next investigate $m(s)$ in the preheat zone, i.e., for $-\infty<s \leq 0$. Substituting expression (5.1) for $\rho^{0}(s)$ in (7.6), we obtain the following relation

$\frac{m(s)-m_{\mathrm{b}}}{m^{0}}=\mathrm{Ka}_{\mathrm{b}}(\theta+1)\left(-\eta(s)+\log \left(\frac{\theta \mathrm{e}^{\eta(s)}+1}{\theta+1}\right)\right)$.

This relation is plotted in Fig. 3 (at the left where $\eta(s)<0$ ) and is similar to the numerical results in Fig. 4. Theoretical and numerical results are almost identical in the complete flame except in the neighborhood of the reaction zone, as expected. If we now take the limit $s \rightarrow-\infty$, we recover relation (6.11), as found from the IA presented in the previous section:

$\frac{m(s)-m_{\mathrm{b}}}{m^{0}} \sim \frac{m^{*}(s)-m_{\mathrm{b}}}{m^{0}}:=K a_{\mathrm{u}}(-\eta(s)-\log (\theta+1))$,

where $m^{*}(s)$ is the linear approximation of $m(s)$ in the preheat zone, indicated by the oblique asymptote in Fig. 3. This means that the mass burning rate $m(s)$ is also linearly varying with $\eta(s)$ in the preheat zone, sufficiently far away from the reaction zone. However, substituting $\eta(s)=0$ in the left-hand side of (7.9), we obtain for the mass burning rate the extrapolated value $m^{*}(0):=m_{\mathrm{b}}-\log (\theta+1) \mathrm{Ka}_{\mathrm{u}} m^{0} \neq m_{\mathrm{b}}$, due to expansion of the gas mixture. If we next replace the mass burning rate $m_{\mathrm{u}}$ by $m\left(s_{\text {ref }}\right)$, and if we take $\left|s_{\text {ref }}\right|$ large enough, we have

$$
\begin{aligned}
\frac{m_{\mathrm{u}}}{m^{0}} & \sim \frac{m_{\mathrm{b}}}{m^{0}}+\left(-\eta_{\mathrm{ref}}-\log (\theta+1)\right) \mathrm{Ka}_{\mathrm{u}}=1-\mathcal{M}_{\mathrm{b}} \mathrm{Ka}_{\mathrm{b}}+\left(-\eta_{\mathrm{ref}}-\log (\theta+1)\right) \mathrm{Ka}_{\mathrm{u}}+\mathcal{O}\left(\mathrm{Ka}_{\mathrm{b}}^{2}\right) \\
& =1-\mathcal{M}_{\mathrm{u}}\left(\eta_{\mathrm{ref}}\right) \mathrm{Ka}_{\mathrm{u}}+\mathcal{O}\left(\mathrm{Ka}_{\mathrm{u}}^{2}\right),
\end{aligned}
$$

implying that the relations (4.6) and (6.9) with the Markstein numbers $\mathcal{M}_{\mathrm{b}}$ and $\mathcal{M}_{\mathrm{u}}\left(\eta_{\text {ref }}\right)$ defined in (5.6) and (6.10), respectively, are consistent with the flamelet model presented Sect. 2. Finally, if we let the flame shrink to a surface, i.e., $\eta_{\text {ref }} \rightarrow 0$, we recover from (7.9) relation (6.11): 
$\frac{m_{\mathrm{u}}-m_{\mathrm{b}}}{m^{0}}=-\log (\theta+1) \mathrm{Ka}_{\mathrm{u}}$

This way, we can define two alternative mass burning rates, one at the reaction zone at $s=0$ and one extrapolated from the unburned gas mixture, and the difference between these is given by (7.11), and only when $\theta=0$ these are equal. Alternatively, we can derive (7.11) from the relations (4.6) and (6.9).

Figure 3 clearly shows that the mass burning rate $m_{\mathrm{b}}$ in the burned gases gives a more accurate representation of the mass burning rate of stretched flames, because the extrapolated value $m^{*}(0)$ deviates considerably from $m(0)$. One should realize, though, that the reaction zone is in reality not infinitely thin. In case of a finite reaction-zone thickness, the value for $m_{\mathrm{b}}$ describes the asymptotic behavior of $m(s)$ in the burned gases, extrapolated to the heart of the reaction zone at $s=0$, e.g., the position of the maximum heat release. In that case, $m_{\mathrm{b}}$ is not exactly on the $m(s)$-curve either because $m(s)$ is not completely linear then for $s>0$. However, $m_{\mathrm{b}}$ is much closer to $m(0)$ than $m^{*}(0)$, because the reaction zone is much thinner than the preheat zone. In other words, the 'error' in the Markstein number $\mathcal{M}_{\mathrm{b}}$ is of the order of $\delta_{\mathrm{r}} / \delta_{\mathrm{f}}=1 / \mathrm{Ze}$, where $\delta_{\mathrm{r}}$ is the reaction-zone thickness, while it is of the order of 1 in the Markstein number of the unburnt gas $\mathcal{M}_{\mathrm{u}}$.

A very important question is what can be learnt from this. First of all, it should be noted that, in general, the laminar burning speed $s_{\mathrm{L}}$ at the unburned flame boundary is used as reference value. For instance, in experiments on spherically expanding flames one often uses Schlieren photography to determine the unburned boundary or another position in the flame to derive $s_{\mathrm{L}}$ or the Markstein number. The above analysis shows that $m_{\mathrm{b}}$ is more appropriate than $m_{\mathrm{u}}$ to predict the mass burning rate in the flame structure. We therefore suggest to use the burned flame boundary instead of the unburned boundary as a reference to determine the mass burning rate in experiments. If another position is preferred for some reason, one should bear in mind that the use of (6.9) and (6.10) could lead to inaccuracies. However, accurate predictions of the mass burning rate $m(s)$ at other positions in the flame structure are possible if we take $m_{\mathrm{b}}$ as reference value and apply the correction suggested by (7.9).

\section{Conclusions}

In this paper we briefly reviewed the flamelet equations for premixed flames, which is a quasi-one-dimensional system of conservation equations formulated in terms of a curvilinear coordinate system moving with the flame. The flamelet equations all contain a source term proportional to the stretch rate, which describes transport along the flame front. Applying the IA to the flamelet equations, we have derived a model relating the mass burning rate in the burned gas mixture to the stretch rate, involving the so-called Karlovitz integrals, which are weighted mass-flow rates. The model is quite generally applicable; the only assumptions we made are, first, the flame is weakly stretched, and second, the reaction zone is infinitely thin.

Next, we have elaborated the model for a flat stagnation flame governed by a one-step irreversible reaction. Herewith we distinguished two cases, viz. with and without thermal expansion. Assuming constant coefficients, we could solve the flamelet equations analytically and determine the exact expressions for the Karlovitz integrals. The resulting expressions for the mass burning rate, and more specifically the Markstein number, reproduce the well-known expressions obtained with LAEA provided $|\mathrm{Le}-1|=\mathcal{O}(1 / \mathrm{Ze})[7,16]$. It should be emphasized, however, that our model does not rely on the assumption that $\mid$ Le $-1 \mid \ll 1$ unlike for LAEA.

Alternatively, in case of thermal expansion, we have also determined a model for the mass burning rate at a reference position in the preheat zone, near the unburned gas mixture. Comparing our result with the expression obtained via LAEA [7] we have one term extra due to the finite thickness of the flame. This means in fact that LAEA uses the location of the reaction zone to determine the mass burning rate in both the burned and unburned gas mixture. Also for our flamelet model, we have two possibilities to define the mass burning rate at the reaction zone, i.e., the mass burning rate $m_{\mathrm{b}}$ at the burned edge of the flame and the mass burning rate $m^{*}(0)$ extrapolated from the unburned gas mixtured. It turns out that first choice is the most accurate.

The flamelet model presented in this paper can be extended as follows. First, it can be applied to determine the mass burning rate of spherically expanding flames. The flamelet equations can still be solved analytically and 
expressions for the Markstein number can be derived. Another possibility would be to apply it to arbitrary flames, but then the flamelet equations have to be solved numerically and the corresponding Karlovitz integrals have to be computed numerically as well. Finally, the model can be combined with the G-equation to determine the propagation of the flame surfaces in combination with a CFD code to compute the flow.

\section{Appendix: The stretch rate of a plane stagnation flame}

In this appendix we derive expressions for the stretch rate $K(s)$ of a plane stagnation flame and show that it is nearly constant in the entire preheat zone and in the burned gas mixture close to the reaction zone, provided the flame is only weakly stretched. Therefore, we restrict ourselves to weakly stretched flames, i.e., the Karlovitz number $K a_{\mathrm{b}} \ll 1$. In this appendix we frequently refer to the parameters $\delta_{\mathrm{f}}, K a_{\mathrm{b}}$ and $\theta$, which are defined by (4.1), (4.2) and (5.1), respectively.

From the momentum equations, describing the two-dimensional stagnation flow in the flame, we can derive the following differential equation for $K[27,28]$ :

$m \frac{\mathrm{d} K}{\mathrm{~d} s}-\frac{\mathrm{d}}{\mathrm{d} s}\left(\mu \frac{\mathrm{d} K}{\mathrm{~d} s}\right)=\rho_{\mathrm{u}} a^{2}-\rho K^{2}$,

where $\mu$ is the dynamic viscosity of the gas mixture and $a$ the applied strain rate at the unburned side of the flame. This equation has to be coupled with the flamelet equation (2.6a), with $\sigma(s)=1$, in order to determine $K(s)$ and $m(s)$. We derive approximate solutions of these equations, separately for the preheat zone and for the burned gas mixture. Let $\ell$ be the stand-off distance, i.e., the stagnation plane is located at $s=\ell$, and assume that $\ell \gg \delta_{\mathrm{f}}$. Applying an appropriate scaling to (9.1) and (2.6a), with length scales $\delta_{\mathrm{f}}$ for the preheat zone and $\ell$ for the burned gas mixture, respectively, we conclude that in the preheat zone the convective and viscous terms are of comparable size whereas the stretch terms in both equations are negligible. On the other hand, in the burned gas mixture, the viscous term is negligible but the stretch terms have to be included. Finally, taking $\mu$ constant, replacing $m(s)$ in the preheat zone by $m^{0}, \rho_{\mathrm{u}}$ by $\rho_{\mathrm{u}}^{0}$ and $\rho(s)$ in the burnt gas by $\rho_{\mathrm{b}}^{0}$, we obtain the following set of equations

$m^{0} \frac{\mathrm{d} K}{\mathrm{~d} s}-\mu \frac{\mathrm{d}^{2} K}{\mathrm{~d} s^{2}}=0, \quad-\infty<s<0$,

$m \frac{\mathrm{d} K}{\mathrm{~d} s}=\rho_{\mathrm{u}}^{0} a^{2}-\rho_{\mathrm{b}}^{0} K^{2}, \quad \frac{\mathrm{d} m}{\mathrm{~d} s}=-\rho_{\mathrm{b}}^{0} K, \quad 0<s<\ell$,

which we have to solve subject to the following boundary conditions

$K(-\infty)=a, \quad \frac{\mathrm{d} K}{\mathrm{~d} s}(\ell)=0, \quad m(\ell)=0$.

Moreover, we require continuity of $K(s), \frac{\mathrm{d} K}{\mathrm{~d} s}(s)$ and $m(s)$ at $s=0$.

The solution of (9.2a), subject to the first boundary condition in $(9.2 \mathrm{c})$, reads

$K(s)=a\left(1+A \mathrm{e}^{\eta(s) / \operatorname{Pr}}\right), \quad \eta(s):=\frac{s}{\delta_{\mathrm{f}}}, \quad-\infty<s<0$,

where $A$ is a coefficient to be determined later and $\operatorname{Pr}:=\mu c_{p} / \lambda$ is the Prandtl number of the gas mixture. The solution of $(9.2 b)$ is more involved. Combining both equations, we obtain the following differential equation for 
the mass burning rate $m$ :

$m \frac{\mathrm{d}^{2} m}{\mathrm{~d} s^{2}}-\left(\frac{\mathrm{d} m}{\mathrm{~d} s}\right)^{2}+\rho_{\mathrm{u}}^{0} \rho_{\mathrm{b}}^{0} a^{2}=0$.

The solution of (9.4), subject to the third boundary condition in (9.2c), can be written in the form $m(s)=$ $-B \sin q \eta(s-\ell)$ with $B, q>0$, provided $B q / \delta_{\mathrm{f}}=a \sqrt{\rho_{\mathrm{u}}^{0} \rho_{\mathrm{b}}^{0}}$. Substituting this expression in the second equation in $(9.2 b)$, we obtain

$K(s)=a \sqrt{\theta+1} \cos q \eta(s-\ell), \quad 0<s<\ell$.

The solution in (9.3) and (9.5) contains three unknown constants, viz. $A, q$ and $\ell$, that we determine from continuity of $K(s), \frac{\mathrm{d} K}{\mathrm{~d} s}(s)$ and $m(s)$ at $s=0$. This gives the following set of nonlinear equations

$1+A=\sqrt{\theta+1} \cos q \eta(\ell), \quad \eta(\ell)=\frac{\ell}{\delta_{\mathrm{f}}}$,

$A=\operatorname{Pr} q \sqrt{\theta+1} \sin q \eta(\ell)$,

$m^{0} q=a \delta_{\mathrm{f}} \rho_{\mathrm{b}}^{0} \sqrt{\theta+1} \sin q \eta(\ell)$.

Using the Taylor series $\sqrt{1+x}=1+\frac{1}{2} x-\frac{1}{8} x^{2}+\mathcal{O}\left(x^{3}\right)$ for $x \rightarrow 0$, we can show that the solution of (9.6), to first order accuracy, is given by

$$
\begin{aligned}
& A=\theta \operatorname{PrKa}_{\mathrm{b}}+\mathcal{O}\left(\mathrm{Ka}_{\mathrm{b}}^{2}\right), \\
& q=\sqrt{\theta} \mathrm{Ka}_{\mathrm{b}}+\mathcal{O}\left(\mathrm{Ka}_{\mathrm{b}}^{2}\right), \\
& \tan \left(\frac{q \ell}{\delta_{\mathrm{f}}}\right)=\sqrt{\theta}+\mathcal{O}\left(\mathrm{Ka}_{\mathrm{b}}^{2}\right) .
\end{aligned}
$$

Combining the solutions in (9.3) and (9.5), we have

$K^{*}(s):=\frac{K(s)-a}{a}=\left\{\begin{array}{l}A \mathrm{e}^{\eta(s) / \mathrm{Pr}}, \quad-\infty<s<0, \\ \sqrt{\theta+1} \cos q(\eta(s)-\ell)-1, \quad 0<s<\ell,\end{array}\right.$

with the parameters $A, q$ and $\ell$ given in (9.7); see Fig. 5. Clearly, the relative deviation of $K(s)$ from $a$ is at most $2 \times 10^{-2}$ in the preheat zone. Note that also in the burned gas mixture, close to the reaction layer, $K^{*}(s)$ is still small. For example, $K^{*}(s)<0.1402$ for $0 \leq \eta(s) \leq 5$. Finally, note that $\ell \gg \delta_{\mathrm{f}}$ indeed, as we assumed in the derivation of (9.2).
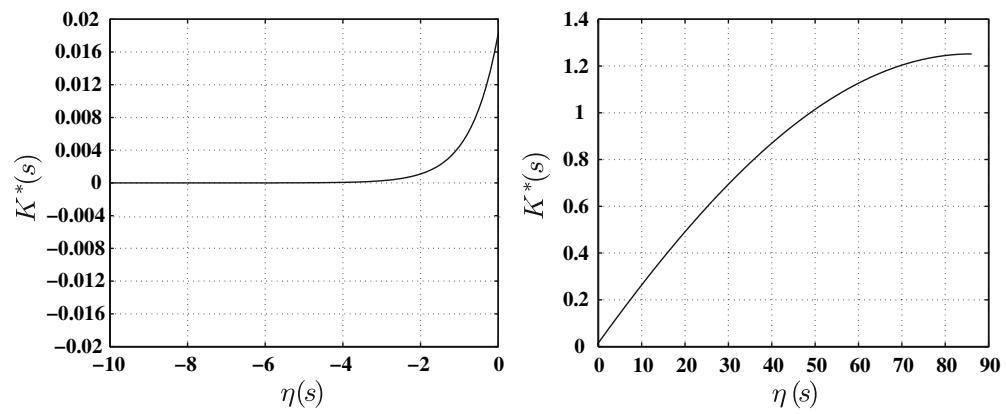

Fig. $5 K^{*}(s)$ of a flame with infinitely thin reaction zone at $\eta(s)=0$, in the preheat zone (left) and in the burned gas mixture (right). Parameter values are: $a=10 \mathrm{~s}^{-1}, \operatorname{Pr}=0.71, \theta=4, \delta_{\mathrm{f}}=6.22 \times 10^{-2} \mathrm{~cm}$ and $\mathrm{Ka}_{\mathrm{b}}=6.4 \times 10^{-3}$ 


\section{References}

1. Karlovitz B, Denniston DW Jr, Knapschaefer DH, Wells FE (1953) Studies on turbulent flames. Fourth symposium on combustion. Williams and Wilkins, Baltimore, pp 613-620

2. Lewis B, von Elbe G (1961) Combustion, flames and explosion of gases. Academic Press, New York

3. Markstein GH (1964) Nonsteady flame propagation. Pergamon Press, Oxford

4. Buckmaster J (1979) The quenching of two-dimensional premixed flames. Acta Astronautica 6:741-769

5. Matalon M (1983) On flame stretch. Combust Sci Technol 31:169-181

6. Matalon M, Matkowsky BJ (1982) Flames as gasdynamic discontinuities. J Fluid Mech 124:239-259

7. Clavin P (1985) Dynamic behavior of premixed flame fronts in laminar and turbulent flows. Prog Energy Combust Sci 11:1-59

8. Clavin P, Williams FA (1982) Effects of molecular diffusion and thermal expansion on the structure and dynamics of premixed flames in turbulent flows of large scale and low intensity. J Fluid Mech 116:251-282

9. Peters N (1992) A spectral closure for premixed turbulent combustion in the flamelet regime. J Fluid Mech 42:611-629

10. Chung SH, Law CK (1988) An integral analysis of the structure of stretched premixed flames. Combust Flame 72:325-336

11. de Goey LPH, Mallens RMM, ten Thije Boonkkamp JHM (1997) An evaluation of different contributions to flame stretch for stationary premixed flames. Combust Flame 110:54-66

12. de Goey LPH, ten Thije Boonkkamp JHM (1999) A flamelet description of premixed laminar flames and the relation with flame stretch. Combust Flame 119:253-271

13. de Goey LPH, ten Thije Boonkkamp JHM (1997) A mass-based definition of flame stretch for flames with finite thickness. Combust Sci Technol 122:399-405

14. ten Thije Boonkkamp JHM, de Goey LPH (1997) Analysis of stretched flames for laminar and turbulent combustion. In: Turbulence, heat and mass transfer 2, Proceedings of the 2nd int. symp. on turbulence, heat and mass transfer. Delft University Press, Delft, pp 709-718

15. ten Thije Boonkkamp JHM, de Goey LPH (1997) Integral analysis of stretched flames. In: Proceedings of the fourth Asian-Pacific international symposium on combustion and energy utilization. Chulalongkorn University, Bangkok, pp 466-474

16. Joulin G, Clavin P (1979) Linear stability analysis of non-adiabatic flames: diffusional-thermal model. Combust Flame 35:139-153

17. van Oijen JA, Bastiaans RJM, Groot GRA, de Goey LPH (2005) Direct numerical simulations of premixed turbulent flames with reduced chemistry: validation and flamelet analysis. Flow, Turbulence Combustion 75(1-4):67-84

18. de Swart JAM, Groot GRA, van Oijen JA, ten Thije Boonkkamp JHM, de Goey LPH (2006) Detailed analysis of the mass burning rate of stretched flames including preferential diffusion effects. Combust Flame 145:245-258

19. Williams FA (1974) A review of some theoretical considerations of turbulent flame structure. Agard Conference Proceedings vol 164, p II1.1

20. Aris R (1962) Vectors, tensors, and the basic equations of fluid mechanics. Dover Publications, Inc., New York

21. ten Thije Boonkkamp JHM, de Goey LPH (1999) A flamelet model for premixed stretched flames. Combust Sci Technol 149:183200

22. Bush WB, Fendell FE (1970) Asymptotic analysis of laminar flame propagation for general Lewis numbers. Combust Sci Technol $1: 421-428$

23. Bastiaans RJM, Martin SM, Pitsch H, van Oijen JA, de Goey LPH (2005) Flamelet analysis of turbulent combustion. In: Lecture Notes in Computer Science Vol. 3516, pp 64-71

24. Barenblatt GI, Zeldovich YaB, Istratov AG (1962) O diffuzionno-teplovoi ustoichivosti laminarnogo plameni (on the diffusionalthermal stability of laminar flames). Prikl Mekh i Tekh Fiz 4:21

25. Williams FA (1985) Combustion theory, 2nd edn. Addison-Wesley, Redwood City

26. Abramowitz M, Stegun IA (eds) (1964) Handbook of mathematical functions. National Bureau of Standards, Washington

27. Dixon-Lewis G (1990) Structure of laminar flames. Proc Combust Inst 23:305-324

28. van Oijen JA (2002) Flamelet-generated manifolds: development and application to premixed laminar flames. PhD. Thesis, Eindhoven University of Technology 